\title{
Actividad Física en Habitantes de 15 a 49 Años de una Localidad de Bogotá, Colombia, 2004
}

\author{
Sonia C. Mantilla-Toloza
}

Fisioterapeuta. M. Sc. Salud Pública. Investigadora independiente.

E-mail: soniacarilina.mantilla@alu.um.es

Recibido 15 Noviembre 2004/Enviado para Modificación 15 Diciembre 2005/Aceptado 10 Octubre 2006

RESUMEN

Objetivo Identificar las características de la práctica de actividad física en cuatro escenarios: el tiempo libre, el trabajo, el transporte y el hogar; los estados de cambio y las barreras al cambio.

Métodos se definió una muestra de 460 personas entre 15 y 49 años, residentes en la Localidad de Teusaquillo, Bogotá, Colombia; se aplicó una encuesta validada internacionalmente que incluía instrumentos específicos para la medición de los estados de cambio y las barreras.

Resultados Se encontraron niveles de actividad física suficiente en el 40 a $46 \%$ de la población estudiada. Los residentes de la Localidad fueron más activos en los escenarios del trabajo y el hogar. En relación con los estados de cambio de la actividad física, la mitad de la población encuestada se encontró en estados previos a la acción, mientras que la otra mitad se clasificó como regularmente activa. Las barreras más frecuentes para la actividad física son la falta de voluntad y la falta de tiempo.

Conclusiones Fue posible identificar las características de la actividad física en una Localidad de Bogotá y algunos de los factores asociados.

Palabras Clave: Actividad motora, promoción de la salud (fuente: DeCS, BIREME).

\section{ABSTRACT}

Physical activity in people aged 15 to 49 living in a particular locality in Bogotá, Colombia, 2004

Objective Identifying the characteristics of practising physical activity in four scenarios (in free-time, at work, type of transport and in the home) as well as the stages of change and barriers against it. 
Methods A sample of 460 people aged 15 to 49 , residing in Teusaquillo, Bogotá, Colombia, was defined. An internationally-validated survey was applied which included specific instruments for measuring the stages of change and barriers against it.

Results Sufficient levels of physical activity were found in $40 \%$ to $46 \%$ of the population being studied. The residents of this particular area were more active at wok and in the home than during free time and when being transported. Half of the population being surveyed were classified as being in stages prior to action (regarding stages of changing physical activity), whilst the other $50 \%$ were regularly active. The most frequently occurring barriers to physical activity were a lack of willpower and lack of time.

Conclusions The characteristics of physical activity could be identified in a specific part of Bogotá; some associated factors were also identified.

Key Words: physical activity, health promotion, chronic disease prevention, Colombia (source: DeCS, BIREME).

L

os indicadores básicos de la situación de salud en Colombia reflejan que la principal causa de mortalidad en mujeres y hombres mayores de 45 años corresponde a las enfermedades crónicas y dentro de éstas, las enfermedades cardio-cerebrovasculares, relacionadas con el sedentarismo y otros factores de riesgo (1-7). En Bogotá, en el año de 1999, ocurrieron 7 296 muertes por enfermedad cardio-cerebrovascular, lo que representa una tasa de 116,2 muertes por cien mil habitantes (8). En la Localidad de Teusaquillo, la tasa de mortalidad por enfermedades cardio-cerebrovasculares es de 180 por cien mil habitantes (9).

Para superar el sedentarismo se requiere la actividad física. La recomendación actual indica que deben acumularse 30 minutos de actividad física moderada cada día $(5,10,11)$.

Estudios descriptivos han caracterizado la actividad física en diferentes poblaciones, estableciendo algunas relaciones con variables sociodemográficas y/o ambientales como factores que podrían favorecerla. Por ejemplo, en Australia cerca de la tercera parte de los adultos son sedentarios en su tiempo libre, lo cual es similar a lo que se reporta en Norteamérica y Nueva Zelanda. Las razones más comunes por las que la gente no se ejercita son la falta de tiempo, la falta de habilidad y la falta de voluntad (4). En Colombia, específicamente en Bogotá, se determinó que sólo el 37 \% de la población entre 18 y 65 años era regularmente activa (12). 
Actualmente se ha considerado que el soporte ambiental, social y la infraestructura de la ciudad o del barrio pueden contribuir a la adopción y mantenimiento de estilos de vida más activos (13-16). Se ha reconocido que la práctica de la actividad física es una herramienta clave para reducir el sedentarismo y por lo tanto la prevalencia de las enfermedades crónicas vasculares (17-24), para lo cual se requiere identificar los niveles de actividad física en comunidades específicas. La presente investigación apuntó a identificar el nivel de actividad física de los habitantes de una Localidad de Bogotá y algunos de los factores asociados.

\section{METODOS}

Se realizó un estudio descriptivo de tipo transversal. La población correspondió a personas entre 15 y 49 años de edad, residentes en la localidad de Teusaquillo de Bogotá, con predominio del estrato socioeconómico 4. Se estimó una muestra probabilística y aleatoria simple de 460 personas y se realizó un muestreo en dos etapas, ubicando los sectores censales y los hogares, según los estratos socioeconómicos.

Se recogió información sobre sexo, edad, estado civil, nivel educativo, afiliación a la seguridad social, percepción del estado de salud, barreras para ser activo y percepción de las condiciones ambientales del barrio. La información sobre actividad física se recogió por medio del "International physical activity questionnaire” (IPAQ). La actividad física se consideró en tres niveles: Insuficientemente activo: quien durante los últimos 7 días registró un gasto calórico menor de $600 \mathrm{METs}^{1} / \mathrm{min} /$ semana; Suficientemente activo: durante los últimos 7 días, registró un gasto calórico entre 600 y 1499 METs/min/semana; Altamente activo: durante los últimos 7 días, registró un gasto calórico superior o igual a $1500 \mathrm{METs} / \mathrm{min} / \mathrm{semana}$.

El sedentarismo se interpretó como poca o ninguna ejecución de actividad física. Se midió como el tiempo promedio semanal que la persona permanece sentada, en un día entre semana, en un día de fin de semana y durante el desplazamiento en un vehículo motorizado durante la semana. Los estados de cambio se consideraron como etapas a través de las cuales una persona puede modificar su comportamiento respecto a la actividad física. Las etapas o estados de cambio se consideran para períodos de seis meses, y son (11): Precontemplación: No hace actividad física y actualmente no tiene

\footnotetext{
${ }^{1}$ METs: Equivalente metabólico. Un METs equivale al gasto energético de 1,2 kcal/min para un individuo de $70 \mathrm{~kg}$, que corresponde al estado de reposo.
} 
la intención de hacerla; Contemplación: Está considerando ser físicamente activo. Tiene la intención de hacerlo; Preparación: Está tratando pero aún no es regularmente activo.

Acción: Es regularmente activo pero por menos de seis meses.

Mantenimiento: Se ha mantenido regularmente activo durante seis meses o más.

Recaída: Hace más de seis meses era regularmente activo, pero ya no lo es. Por medio del programa STATA v.5, se estableció la prevalencia y los niveles de actividad física; se estimaron las razones de prevalencia para identificar las relaciones entre las variables consideradas y la actividad física en los contextos trabajo, hogar, tiempo libre y transporte.

\section{RESULTADOS}

Se obtuvo información completa de 453 personas, de las cuales el 78 \% pertenecían a hogares del estrato 4 y el resto al estrato 3.

Características ambientales

El 90 \% de las personas consideraron que su barrio tenía una buena infraestructura de establecimientos comerciales, paraderos de bus y ciclo rutas cercanas, así como dotación de sitios recreacionales. El 62,3 \% consideró que había mucho tráfico en las calles, lo que dificultaba caminar en el barrio, pero el 69,5 \% estimó que existían muchos aspectos interesantes para mirar mientras se caminaba por el barrio. En cuanto a la seguridad, el 64,1 \% consideró que su barrio era inseguro para caminar en el día, y 61,7 \% en la noche.

Barreras para la actividad física

Se identificaron como barreras principales para la actividad física la falta de voluntad, $41,1 \%$ y la falta de tiempo, $40,4 \%$.

\section{Sedentarismo}

El promedio de tiempo que las personas encuestadas permanecían sentadas era de 2325 minutos/semana, es decir, unas 5 horas diarias.

Niveles de actividad física

Las frecuencias correspondientes a los niveles de actividad física en los contextos tiempo libre, trabajo, hogar y transporte, según las características 
sociodemográficas y barreras para ser físicamente activo, se muestran en la Tabla 1. Se encontraron diferencias significativas en los siguientes ámbitos y características.

Actividad física en el tiempo libre: Hombres, jóvenes, personas que pertenecían al estrato 4, mayor nivel educativo, estudiantes, ingresos mayores a 3 salarios mínimos legales vigentes (SMLV) y en los que consideran que su estado de salud es muy bueno.

Actividad física en el trabajo: Hombres, personas mayores de 20 años, mayor nivel educativo, unión estable, empleadores, personas de menores ingresos y quienes perciben que su estado de salud es bueno o muy bueno.

Actividad física en el hogar: Mujeres, personas con edades entre 40 y 49 años, empleados, nivel educativo y socioeconómico bajo, personas no afiliadas a la seguridad social y aquellas que perciben su estado de salud regular y malo.

Actividad física en el transporte: Hombres, jóvenes, estudiantes, personas sin unión estable, los que no estaban afiliados a la seguridad social y los que consideran que su estado de salud es muy bueno.

\section{Estados de cambio respecto a la actividad física}

Se obtuvo información de 380 personas. Del total de la población estudiada, 190 personas se encontraron en estados previos a la acción. El 50 \% restante, se clasificó en el grupo de acción o en el de mantenimiento. La distribución en la muestra de las proporciones por cada etapa de cambio fue como sigue: precontemplación $14,7 \%$, contemplación $28,4 \%$, preparación 6,8 \%, acción $3,2 \%$ y mantenimiento $46,8 \%$.

Se apreció mayor inactividad física a medida que aumentaba la edad de la población encuestada, en el estrato 3 , en personas con menores ingresos, en los empleados, en quienes se encontraban afiliados al sistema de seguridad social y en las personas para quienes la falta de tiempo, de energía, de voluntad y de recursos son barreras para ser físicamente activos.

Un 30,4 \% de las personas en precontemplación se encontraban en recaída. Lo mismo ocurrió con el 60,7 \% de las personas en contemplación. 
Tabla 1. Niveles de actividad física

\begin{tabular}{|c|c|c|c|c|c|c|c|c|c|c|c|c|c|c|c|}
\hline \multirow{2}{*}{\multicolumn{2}{|c|}{ Características }} & \multicolumn{2}{|c|}{ Total } & \multicolumn{3}{|c|}{ Tiempo libre \% } & \multicolumn{3}{|c|}{ Hogar \% } & \multicolumn{3}{|c|}{ Transporte \% } & \multicolumn{3}{|c|}{ Trabajo \% } \\
\hline & & $\mathrm{N}^{0}$ & $\%$ & 1 & $\mathrm{~s}$ & A & 1 & $\mathrm{~s}$ & A & 1 & $\mathrm{~s}$ & A & 1 & $\mathrm{~s}$ & A \\
\hline \multirow{3}{*}{ Sexo } & Femenino & 256 & 56,5 & 63,3 & 21,9 & 14,8 & 37,5 & 24,2 & 38,3 & 64,8 & 27,7 & 7,4 & 61,3 & 10,9 & 27,7 \\
\hline & Masculino & 197 & 43,5 & 52,8 & 21,8 & 25,4 & 74,6 & 14,7 & 10,7 & 53,8 & 35,5 & 10,7 & 47,7 & 24,9 & 27,4 \\
\hline & Total & 453 & 100,0 & 58,7 & 21,9 & 19,4 & 53,6 & 20,1 & 26,3 & 60,0 & 31,1 & 8,8 & 55,4 & 17,0 & 27,6 \\
\hline \multirow{5}{*}{ Edad } & 15 a 19 años & 45 & 9,9 & 46,7 & 26,7 & 26,7 & 60,0 & 20,0 & 20,0 & 44,4 & 46,7 & 8,9 & 68,9 & 26,7 & 4,4 \\
\hline & 20 a 29 años & 101 & 22,3 & 57,4 & 19,8 & 22,8 & 56,4 & 19,8 & 23,8 & 53,5 & 36,6 & 9,9 & 51,5 & 14,9 & 33,7 \\
\hline & 30 a 39 años & 105 & 23,2 & 60,0 & 25,7 & 14,3 & 57,1 & 20,0 & 22,9 & 57,1 & 34,3 & 8,6 & 54,3 & 16,2 & 29,5 \\
\hline & 40 a 49 años & 202 & 44,6 & 61,4 & 19,8 & 18,8 & 49,0 & 20,3 & 30,7 & 68,3 & 23,3 & 8,4 & 55,0 & 16,3 & 28,7 \\
\hline & Total & 453 & 100,0 & 58,7 & 21,9 & 19,4 & 53,6 & 20,1 & 26,3 & 60,0 & 31,1 & 8,8 & 55,4 & 17,0 & 27,6 \\
\hline \multirow{3}{*}{$\begin{array}{l}\text { Estado } \\
\text { Civil }\end{array}$} & No unido & 232 & 51,2 & 58,2 & 18,5 & 23,3 & 56,5 & 19,4 & 24,1 & 53,4 & 37,1 & 9,5 & 58,2 & 17,7 & 24,1 \\
\hline & Unido & 221 & 48,8 & 59,3 & 25,3 & 15,4 & 50,7 & 20,8 & 28,5 & 67,0 & 24,9 & 8,1 & 52,5 & 16,3 & 31,2 \\
\hline & Total & 453 & 100,0 & 58,7 & 21,9 & 19,4 & 53,6 & 20,1 & 26,3 & 60,0 & 31,1 & 8,8 & 55,4 & 17,0 & 27,6 \\
\hline \multirow{3}{*}{ Estrato } & 3 & 101 & 22,3 & 68,3 & 15,8 & 15,8 & 38,6 & 15,8 & 45,5 & 63,4 & 30,7 & 5,9 & 53,5 & 12,9 & 33,7 \\
\hline & 4 & 352 & 77,7 & 56,0 & 23,6 & 20,5 & 58,0 & 21,3 & 20,7 & 59,1 & 31,3 & 9,7 & 56,0 & 18,2 & 25,9 \\
\hline & Total & 453 & 100,0 & 58,7 & 21,9 & 19,4 & 53,6 & 20,1 & 26,3 & 60,0 & 31,1 & 8,8 & 55,4 & 17,0 & 27,6 \\
\hline \multirow{4}{*}{$\begin{array}{c}\text { Nivel } \\
\text { Educativo }\end{array}$} & Básica & 151 & 33,3 & 62,9 & 19,2 & 17,9 & 41,1 & 19,9 & 39,1 & 58,9 & 30,5 & 10,6 & 57,6 & 11,9 & 30,5 \\
\hline & Superior & 300 & 66,2 & 56,7 & 23,0 & 20,3 & 59,7 & 20,3 & 20,0 & 60,3 & 31,7 & 8,0 & 54,7 & 19,7 & 25,7 \\
\hline & NS/NR & 2 & 0,4 & 50,0 & 50,0 & 0,0 & 100,0 & 0,0 & 0,0 & 100,0 & 0,0 & 0,0 & 0,0 & 0,0 & 100,0 \\
\hline & Total & 453 & 100,0 & 58,7 & 21,9 & 19,4 & 53,6 & 20,1 & 26,3 & 60,0 & 31,1 & 8,8 & 55,4 & 17,0 & 27,6 \\
\hline \multirow{4}{*}{ Ocupación } & Empleador & 177 & 39,1 & 53,1 & 22,6 & 24,3 & 55,4 & 20,9 & 23,7 & 62,1 & 29,9 & 7,9 & 50,8 & 13,6 & 35,6 \\
\hline & Empleado & 193 & 42,6 & 67,9 & 21,2 & 10,9 & 50,8 & 18,1 & 31,1 & 67,4 & 25,4 & 7,3 & 57,0 & 16,6 & 26,4 \\
\hline & Estudiante & 81 & 17,9 & 49,4 & 22,2 & 28,4 & 58,0 & 21,0 & 21,0 & 39,5 & 46,9 & 13,6 & 60,5 & 25,9 & 13,6 \\
\hline & Total & 453 & 100,0 & 58,7 & 21,9 & 19,4 & 53,6 & 20,1 & 26,3 & 60,0 & 31,1 & 8,8 & 55,4 & 17,0 & 27,6 \\
\hline
\end{tabular}




\begin{tabular}{|c|c|c|c|c|c|c|c|c|c|c|c|c|c|c|c|}
\hline & & \multicolumn{2}{|c|}{ Total } & \multicolumn{3}{|c|}{ Tiempo libre \% } & \multicolumn{3}{|c|}{ Hogar $\%$} & \multicolumn{3}{|c|}{ Transporte \% } & \multicolumn{3}{|c|}{ Trabajo \% } \\
\hline \multicolumn{2}{|c|}{ Características } & $\mathrm{N}^{\circ}$ & $\%$ & 1 & $\mathrm{~s}$ & A & 1 & $\mathrm{~s}$ & A & 1 & $\mathrm{~S}$ & A & 1 & $\mathrm{~s}$ & A \\
\hline \multirow{5}{*}{ Ingresos } & Hasta 3 smlv & 126 & 27,8 & 65,9 & 17,5 & 16,7 & 40,5 & 24,6 & 34,9 & 56,3 & 34,9 & 8,7 & 46,0 & 11,1 & 42,9 \\
\hline & 3 a 4 & 94 & 20,8 & 53,2 & 24,5 & 22,3 & 51,1 & 19,1 & 29,8 & 57,4 & 27,7 & 14,9 & 59,6 & 14,9 & 25,5 \\
\hline & mas de 4 & 209 & 46,1 & 56,9 & 23,4 & 19,6 & 64,1 & 18,7 & 17,2 & 60,3 & 33,0 & 6,7 & 59,3 & 22,0 & 18,7 \\
\hline & NS/NR & 24 & 5,3 & 58,3 & 20,8 & 20,8 & 41,7 & 12,5 & 45,8 & 87,5 & 8,3 & 4,2 & 54,2 & 12,5 & 33,3 \\
\hline & Total & 453 & 100,0 & 58,7 & 21,9 & 19,4 & 53,6 & 20,1 & 26,3 & 60,0 & 31,1 & 8,8 & 55,4 & 17,0 & 27,6 \\
\hline \multirow{4}{*}{$\begin{array}{l}\text { Percepción } \\
\text { Salud }\end{array}$} & Muy bueno & 86 & 19,0 & 41,9 & 22,1 & 36,0 & 59,3 & 20,9 & 19,8 & 51,2 & 34,9 & 14,0 & 57,0 & 17,4 & 25,6 \\
\hline & Bueno & 317 & 70,0 & 60,9 & 22,4 & 16,7 & 52,7 & 19,2 & 28,1 & 60,6 & 31,2 & 8,2 & 53,9 & 18,3 & 27,8 \\
\hline & Regular y malo & 50 & 11,0 & 74,0 & 18,0 & 8,0 & 50,0 & 24,0 & 26,0 & 72,0 & 24,0 & 4,0 & 62,0 & 8,0 & 30,0 \\
\hline & Total & 453 & 100,0 & 58,7 & 21,9 & 19,4 & 53,6 & 20,1 & 26,3 & 60,0 & 31,1 & 8,8 & 55,4 & 17,0 & 27,6 \\
\hline \multirow{4}{*}{$\begin{array}{l}\text { Afiliación } \\
\text { Salud }\end{array}$} & $\mathrm{Si}$ & 396 & 87,4 & 57,8 & 22,5 & 19,7 & 55,8 & 19,9 & 24,2 & 61,1 & 31,1 & 7,8 & 55,1 & 18,2 & 26,8 \\
\hline & No & 44 & 9,7 & 59,1 & 20,5 & 20,5 & 36,4 & 25,0 & 38,6 & 47,7 & 34,1 & 18,2 & 47,7 & 11,4 & 40,9 \\
\hline & $N S / N R$ & 13 & 2,9 & 84,6 & 7,7 & 7,7 & 46,2 & 7,7 & 46,2 & 69,2 & 23,1 & 7,7 & 92,3 & 0,0 & 7,7 \\
\hline & Total & 453 & 100,0 & 58,7 & 21,9 & 19,4 & 53,6 & 20,1 & 26,3 & 60,0 & 31,1 & 8,8 & 55,4 & 17,0 & 27,6 \\
\hline \multirow{3}{*}{$\begin{array}{c}\text { Barrera } \\
\text { Falta de } \\
\text { tiempo }\end{array}$} & No & 270 & 59,6 & 43,0 & 27,4 & 29,6 & 54,1 & 22,6 & 23,3 & 54,8 & 35,2 & 10,0 & 58,9 & 19,3 & 21,9 \\
\hline & $\mathrm{Si}$ & 183 & 40,4 & 82,0 & 13,7 & 4,4 & 53,0 & 16,4 & 30,6 & 67,8 & 25,1 & 7,1 & 50,3 & 13,7 & 36,1 \\
\hline & Total & 453 & 100,0 & 58,7 & 21,9 & 19,4 & 53,6 & 20,1 & 26,3 & 60,0 & 31,1 & 8,8 & 55,4 & 17,0 & 27,6 \\
\hline \multirow{3}{*}{$\begin{array}{l}\text { Barrera } \\
\text { Falta de } \\
\text { voluntad }\end{array}$} & No & 267 & 58,9 & 42,7 & 29,2 & 28,1 & 54,7 & 22,5 & 22,8 & 53,9 & 36,0 & 10,1 & 55,8 & 17,2 & 27,0 \\
\hline & $\mathrm{Si}$ & 186 & 41,1 & 81,7 & 11,3 & 7,0 & 52,2 & 16,7 & 31,2 & 68,8 & 24,2 & 7,0 & 54,8 & 16,7 & 28,5 \\
\hline & Total & 453 & 100,0 & 58,7 & 21,9 & 19,4 & 53,6 & 20,1 & 26,3 & 60,0 & 31,1 & 8,8 & 55,4 & 17,0 & 27,6 \\
\hline \multirow{3}{*}{$\begin{array}{l}\text { Barrera } \\
\text { Falta de } \\
\text { energía }\end{array}$} & No & 360 & 79,5 & 51,1 & 25,6 & 23,3 & 52,5 & 20,0 & 27,5 & 55,8 & 33,9 & 10,3 & 56,4 & 18,6 & 25,0 \\
\hline & $\mathrm{Si}$ & 93 & 20,5 & 88,2 & 7,5 & 4,3 & 58,1 & 20,4 & 21,5 & 76,3 & 20,4 & 3,2 & 51,6 & 10,8 & 37,6 \\
\hline & Total & 453 & 100,0 & 58,7 & 21,9 & 19,4 & 53,6 & 20,1 & 26,3 & 60,0 & 31,1 & 8,8 & 55,4 & 17,0 & 27,6 \\
\hline
\end{tabular}


Relaciones entre variables sociodemográficas, ambientales, barreras y nivel de actividad física

Actividad física en el tiempo libre. Entre otros, el sexo masculino, el estrato, la ocupación y la autopercepción del estado de salud son las variables sociodemográficas que se asociaron directamente con la actividad física en el tiempo libre (Tabla 2).

Tabla 2. Relación: variables independientes vs. actividad física en el tiempo libre

\begin{tabular}{|c|c|c|c|c|}
\hline Característica & $n$ & $\begin{array}{c}\text { Razón } \\
\text { Prevalencia }\end{array}$ & IC 95\% & $\mathrm{P}$ \\
\hline \multicolumn{5}{|l|}{ Sexo } \\
\hline Femenino & 256 & 1 & & \\
\hline Masculino & 197 & 1.29 & $1.03-1.59$ & 0.02 \\
\hline \multicolumn{5}{|l|}{ Ocupación } \\
\hline Empleador & 177 & 1.45 & $1.13-1.9$ & 0.003 \\
\hline Empleado & 193 & 1 & & \\
\hline Estudiante & 81 & 1.57 & $1.17-2.12$ & 0.003 \\
\hline \multicolumn{5}{|l|}{ Percepción salud } \\
\hline Muy bueno & 86 & 2.23 & $1.36-3.7$ & 0.0003 \\
\hline Bueno & 317 & 1.5 & $0.92-2.44$ & 0.07 \\
\hline Regular y malo & 50 & 1 & & \\
\hline \multicolumn{5}{|l|}{ Ambiente } \\
\hline Inseguridad noche & 267 & 0.76 & $0.61-0.94$ & 0.01 \\
\hline Inseguridad dia & 161 & 0.79 & $0.62-1$ & 0.05 \\
\hline \multicolumn{5}{|l|}{ Barreras } \\
\hline Falta de tiempo & 183 & 0.31 & $0.23-0.44$ & 0 \\
\hline Falta de recursos & 43 & 0.54 & $0.31-0.94$ & 0.011 \\
\hline Falta de voluntad & 186 & 0.32 & $0.23-0.44$ & 0 \\
\hline Miedo a lesionarse & 14 & 0.16 & $0.044-0.63$ & 0.0084 \\
\hline Influencia social & 68 & 0.28 & $0.16-0.53$ & 0 \\
\hline Falta de energía & 93 & 0.24 & $0.14-0.44$ & 0 \\
\hline
\end{tabular}

Actividad física en el trabajo. Con relación a la actividad física en el trabajo se encontró una relación significativa con el sexo masculino y asociación inversa con los ingresos mensuales.

Actividad física en el hogar. Entre otros, el sexo masculino, el estrato, nivel educativo, los ingresos mensuales y la afiliación a la seguridad social se asociaron con la actividad física en el hogar (Tabla 3). 
Tabla 3. Relación variables independientes vs. actividad física en el hogar

\begin{tabular}{|c|c|c|c|c|}
\hline Característica & $\mathrm{N}^{0}$ & $\mathrm{RP}$ & IC 95\% & $p$ \\
\hline \multicolumn{5}{|l|}{ Sexo } \\
\hline Femenino & 256 & 1 & & \\
\hline Masculino & 197 & 0.41 & $0.31-0.53$ & 0 \\
\hline \multicolumn{5}{|l|}{ Estrato } \\
\hline 3 & 101 & 1 & & \\
\hline 4 & 352 & 0.69 & $0.56-0.83$ & 0.0006 \\
\hline \multicolumn{5}{|l|}{ Nivel educativo } \\
\hline Básica & 151 & 1 & & \\
\hline Superior & 300 & 0.68 & $0.57-0.83$ & 0.0002 \\
\hline \multicolumn{5}{|l|}{ Ingresos } \\
\hline Hasta 3 smv & 126 & 1 & & \\
\hline 3 a 4 SMV & 94 & 0.82 & $0.64-1.05$ & 0.11 \\
\hline Mas de 4 SMV & 209 & 0.6 & $0.48-0.76$ & 0 \\
\hline \multicolumn{5}{|l|}{ Ambiente } \\
\hline Inseguridad noche & 267 & 1.25 & $1.005-1.56$ & 0.03 \\
\hline Inseguridad día & 161 & 1.54 & $1.27-1.9$ & 0 \\
\hline $\begin{array}{l}\text { Tráfico que dificulta } \\
\text { caminar }\end{array}$ & 281 & 1.4 & $1.12-1.8$ & 0.0011 \\
\hline
\end{tabular}

Actividad física en el transporte. Las variables sociodemográficas que se asociaron directa y significativamente con la actividad física como medio de transporte fueron el sexo masculino, ser estudiante y auto percibir que el estado de salud es muy bueno. El estado civil unido, el grupo de 40 a 49 años de edad, algunas barreras como influencia social, falta de tiempo, falta de voluntad y de energía se asociaron también con la actividad física

\section{DISCUSIÓN}

Una investigación realizada en Bogotá revisó los estados de cambio y obtuvo resultados semejantes a los de esta investigación (25); la mitad de la población estudiada se encontraba en estados de precontemplación, contemplación o preparación para la actividad física. La mayoría de estudios de prevalencia de la actividad física la han examinado en el tiempo libre o como una actividad de tipo recreativo. En relación con ellos, los hallazgos de este trabajo son similares (3,25-31). Pero este estudio aporta que más del $50 \%$ de las personas son insuficientemente activas en los contextos de trabajo, transporte, hogar y tiempo libre. 
La conclusión de que los hombres son más activos en el tiempo libre (58 $\%)$ que las mujeres (42\%) coincide con los resultados de otros estudios (12,29-32). Esta diferencia puede deberse a los distintos roles de los géneros, según las normas sociales y culturales prevalecientes. Como lo han indicado diversos autores, el estrato, los ingresos y el nivel educativo están directamente relacionados con la práctica de la actividad física en el tiempo libre $(12,13,25-28)$. En el presente estudio se encontraron también esas relaciones, además de la autopercepción del estado de salud. Probablemente las personas con mejores condiciones de vida tienen mas facilidades para la actividad física en su tiempo libre. Aunque en otros estudios el incremento de la edad se relaciona con disminución de la actividad física en el tiempo libre, $(12,13,26,29,30)$ en el presente estudio no se encontró relación significativa. Sin embargo, los resultados sugieren que los más jóvenes son más activos que los de mayor edad.

Las barreras más importantes para la actividad física detectadas en este estudio fueron la falta de voluntad y la falta de tiempo. Este hallazgo coincide con un estudio realizado en Perú (26). Respecto a las variables ambientales no se encontraron relaciones con la actividad física. Estos resultados no coinciden con los hallazgos de otros estudios en los que se ha reportado la influencia positiva que tiene el soporte contextual en la predisposición hacia la actividad física (14). Sin embargo, la "inseguridad de día y de noche" se relacionó inversamente con la actividad física en el tiempo libre y "existen cosas interesantes para mirar mientras camina por el barrio" se relacionó directamente. Es por esto que tales aspectos se consideran como parte del soporte social para la promoción de la actividad física (15).

En cuanto a las asociaciones encontradas en la actividad física durante el trabajo, podría considerarse que los hombres tienen ocupaciones que requieren de un gasto calórico mayor, especialmente los de menos ingresos. Las variables que de alguna manera favorecieron la actividad física en el tiempo libre, no operaron de la misma manera que en el hogar. Puede suponerse que las mujeres con menores ingresos, bajo nivel educativa, no afiliadas al sistema de seguridad social, son quienes con mayor frecuencia desempeñan tareas de mantenimiento de la casa. Adicionalmente, la inseguridad en el barrio y el tráfico en las calles podrían motivar a que las amas de casa u otras personas de la familia prefieran hacer actividad física dentro del hogar.

Debido a que el estudio se concentró en una Localidad de Bogotá, los grupos de edad de 15 a 49 años y los estratos socioeconómicos 3 y 4 , los resultados no pueden ser generalizados a otras Localidades con características diferentes. El acercamiento a la comprensión de los niveles de actividad física, los 
estados de cambio y las barreras, puede ser útil para el diseño de otros estudios e intervenciones dirigidas a acrecentar la actividad física •

\section{REFERENCIAS}

1. Lenfant C. Can we prevent cardiovascular diseases in low and middle income countries? Bulletin of the world Health Organization. 2001; 79 (10): 980985.

2. Nissinen A, Berrios X, Puska P. Community- based noncomunicable disease interventions: lessons from developed countries for developing ones. Bulletin of the world Health Organization. 2001, 79 (10):963-970.

3. Organización Panamericana de la Salud. La salud en las Américas. Vol. I. Washington, D.C.: OPS. Publicación científica No 569; 1998.

4. Owen N. Strategic initiatives to promote participation in physical activity. Health Promotion International. 1996;11 (3): 213-218.

5. Physical activity and health: a report of the Surgeon General. Atlanta, Georgia, USA: CDC; 1996.

6. DeJong G. The cost of being couch potato. Mich Health Hosp 2003; 39 (4):2427.

7. Jacobi E, Bull F, Neiman A. Cambios acelerados del estilo de vida obligan a fomentar la Actividad Física como prioridad en la región de las Américas. Revista Panamericana de Salud Pública. 2003; 14 (4): 223-225.

8. Organización Panamericana de la Salud. Ministerio de Salud de Colombia. Instituto Nacional de Salud. Situación de Salud en Colombia. Indicadores básicos. 2002.

9. Secretaría de Salud. Alcaldía Mayor de Bogotá. Mortalidad por residencia Bogotá y Localidades 1998- 2002; 2003.

10. Pate R, Pratt M, Blair SN. Physical Activity and Public health: a recommendation from the Centers for Disease Control and Prevention and the American College of Sports Medicine. Journal of the American Medical Association. 1995;273:402-407.

11. U.S. Department of Health and Human Services. Promoting Physical Activity. A guide for community action. Human Kinetics. United States of America; 1999.

12. Gomez LF. Estudio para determinar los niveles de actividad física en las personas de 18 a 65 años de la ciudad de Bogota. Bogota: Fundación FES SocialInstituto Distrital de Recreación y Deporte; 2003.

13. Salinas J, Vio F. Promoción de la salud y actividad física en Chile: política prioritaria. Revista Panamericana de Salud Pública 2003; 14 (4): 281-288.

14. Baker EA, Brenan LK, Brownson R, Houseman RA. Measuring the determinants of physical activity in the community: current and future directions. Research Quarterly for exercise and sport. 2000; 71 (2): 146-158.

15. Simons-Morton DB, Simons-Morton BG, Parcel GS, Bunker JF. Influencing personal and environmental conditions for community health: a multilevel intervention model. Community health. 1988; 11:25-35. 
16. Addy C, Wilson D, Kirtland K. Associations of perceived social and physical environmental supports with physical activity and walking behavior. American journal of public health 2004; 94 (3): 440-443.

17. Roberts WC. American Journal of cardiology. 1984; 53:261-262.

18. Bronner L, Kanter D, Manson J. Primary prevention of stroke. New England Journal Medicine. 1995, 333: 1392-1400.

19. Evenson K, Stevens J. Effect of cardiorespiratory fitness on mortality among hipertensive and normotensive women and men. Epidemiology 2004; 15 (5): 565-572.

20. Tuomiletho J, Lindstrom J, Eriksson J. Prevention of type 2 diabetes mellitus by changes in lifestyle among subjects with impaired glucose tolerance. New England Journal Medicine. 2001; 344: 1343-1350.

21. Snow HC, Marcus R. Exercise, bone mineral density and osteoporosis. Medicine and science in Sport and Exercise Rev. 1991; 19: 351-388.

22. Luoto R, Latikka P. The effect of physical activity on breast cancer risk: a cohort study of 30.548 women. European Journal Epidemiology. 2000; 16: 973980.

23. Elley R, Kerse N, Arroll B, Robinson E. Effectiveness of counselling patients on physical activity in general practice: cluster randomised controlled trial. BMJ. 2003; 326: 793-796.

24. Byrne A, Byrne DG. The effect of exercise on depression, anxiety and other states: a review. Psychosom Journal Res. 1993; 37: 565-574.

25. Cabrera G, Gomez L, Mateus J. Actividad física y etapas de cambio comportamental en Bogotá. Colombia Medica. 2004; 35: 82-86.

26. Seclen-Palacin J, Jacobi E. Factores sociodemográficos y ambientales asociados con la actividad física deportiva en la población urbana del Perú. Revista Panamericana de Salud Pública. 2003; 14(4): 255-264.

27. Salles R, Werneck G. The association between socio-demographic factors and leisure- time physical activity in the Pro-Saúde study. Cad. Saúde Pública. 2003; 19(4):1095-1105.

28. Martínez JW. Prevalencia de actividad física en Risaralda. Resultados preliminares. Pereira: Fundación Universitaria del Área Andina; 2002.

29. Quintero M. Informe de gestión medicina deportiva comunitaria. "Por su salud muévase pues”. Medellín: Indeportes Antioquia y Dirección Seccional de Salud de Antioquia; 2003.

30. González M, García I, Prieto F. Factores de riesgo de enfermedades crónicas no transmisibles, Valledupar, Colombia. Informe quincenal epidemiológico nacional. Instituto Nacional de Salud, Bogotá, D.C. 2004; 9 (4): 49-62.

31. Cabrera G, Gomez L, Mateus J. Leisure time physical activity among women in a neighborhood in Bogotá, Colombia: prevalence and socio-demographic correlates. Cad. Saúde Pública 2004; 20(4): 1103-1109.

32. Craig CL, Marshall AL, Sjostrom M, Bauman AE, Booth ML, Ainsworth BE et al. International physical activity questionnaire: 12-country reliability and validity. Med Sci Sports Exerc 2003 August; 35(8): 1381-95. 